\title{
Mortality and other adverse outcomes in patients with type 2 diabetes mellitus admitted for COVID-19 in association with glucose-lowering drugs: a nationwide cohort study
}

Luis M. Pérez-Belmonte ${ }^{1 *}$, José David Torres-Peñaa ${ }^{2,3}$, María D. López-Carmona¹, M. Mar. Ayala-Gutiérrez', Francisco Fuentes-Jiménez ${ }^{2,3}$, Lucía Jorge Huerta ${ }^{4}$, Jaime Alonso Muñoz ${ }^{5}$, Manuel Rubio-Rivas ${ }^{6}$, Manel Madrazo ${ }^{7}$, Marcos Guzmán Garcia ${ }^{8}$, Beatriz Vicente Montes ${ }^{9}$, Joaquim Fernández Sola ${ }^{10}$, Javier Ena ${ }^{11}$, Ruth Gonzalez Ferrer ${ }^{12}$, Carmen Mella Pérez ${ }^{13}$, Carlos Jorge Ripper ${ }^{14}$, Jose Javier Napal Lecumberri ${ }^{15}$, Iris El Attar Acedo ${ }^{16}$, Susana Plaza Canteli ${ }^{17}$, Sara Fuente Cosío ${ }^{18}$, Francisco Amorós Martínez ${ }^{19}$, Begoña Cortés Rodríguez ${ }^{20}$, Pablo Pérez-Martínez 2,3* , José Manuel Ramos-Rincón ${ }^{21}$, Ricardo Gómez-Huelgas ${ }^{1}$ and for the SEMI-COVID-19 Network

\footnotetext{
Abstract

Background: Limited evidence exists on the role of glucose-lowering drugs in patients with COVID-19. Our main objective was to examine the association between in-hospital death and each routine at-home glucose-lowering drug both individually and in combination with metformin in patients with type 2 diabetes mellitus admitted for COVID-19. We also evaluated their association with the composite outcome of the need for ICU admission, invasive and non-invasive mechanical ventilation, or in-hospital death as well as on the development of in-hospital complications and a long-time hospital stay.

(Continued on next page)
}

\footnotetext{
* Correspondence: luismiguelpb1984@gmail.com; pablopermar@yahoo.es A complete list of the SEMI-COVID-19 Network members is provided in the Appendix.

${ }^{1}$ Internal Medicine Department, Regional University Hospital of Málaga,

Biomedical Research Institute of Málaga (IBIMA), University of Málaga (UMA),

Avenida de Carlos Haya, s/n, 29010 Málaga, Spain

${ }^{2}$ Lipids and Atherosclerosis Unit, Department of Internal Medicine,

Maimonides Biomedical Research Institute of Cordoba (IMIBIC), Reina Sofia

University Hospital, University of Cordoba, Córdoba, Spain

Full list of author information is available at the end of the article
}

(C) The Author(s). 2020 Open Access This article is licensed under a Creative Commons Attribution 4.0 International License, which permits use, sharing, adaptation, distribution and reproduction in any medium or format, as long as you give appropriate credit to the original author(s) and the source, provide a link to the Creative Commons licence, and indicate if changes were made. The images or other third party material in this article are included in the article's Creative Commons licence, unless indicated otherwise in a credit line to the material. If material is not included in the article's Creative Commons licence and your intended use is not permitted by statutory regulation or exceeds the permitted use, you will need to obtain permission directly from the copyright holder. To view a copy of this licence, visit http://creativecommons.org/licenses/by/4.0/ The Creative Commons Public Domain Dedication waiver (http://creativecommons.org/publicdomain/zero/1.0/) applies to the data made available in this article, unless otherwise stated in a credit line to the data. 
(Continued from previous page)

Methods: We selected all patients with type 2 diabetes mellitus in the Spanish Society of Internal Medicine's registry of COVID-19 patients (SEMI-COVID-19 Registry). It is an ongoing, observational, multicenter, nationwide cohort of patients admitted for COVID-19 in Spain from March 1, 2020. Each glucose-lowering drug user was matched with a user of other glucose-lowering drugs in a 1:1 manner by propensity scores. In order to assess the adequacy of propensity score matching, we used the standardized mean difference found in patient characteristics after matching. There was considered to be a significant imbalance in the group if a standardized mean difference $>10 \%$ was found. To evaluate the association between treatment and study outcomes, both conditional logit and mixed effect logistic regressions were used when the sample size was $\geq 100$.

Results: A total of 2666 patients were found in the SEMI-COVID-19 Registry, 1297 on glucose-lowering drugs in monotherapy and 465 in combination with metformin. After propensity matching, 249 patients on metformin, 105 on dipeptidyl peptidase-4 inhibitors, 129 on insulin, 127 on metformin/dipeptidyl peptidase-4 inhibitors, 34 on metformin/sodium-glucose cotransporter 2 inhibitor, and 67 on metformin/insulin were selected. No at-home glucose-lowering drugs showed a significant association with in-hospital death; the composite outcome of the need of intensive care unit admission, mechanical ventilation, or in-hospital death; in-hospital complications; or long-time hospital stays.

Conclusions: In patients with type 2 diabetes mellitus admitted for COVID-19, at-home glucose-lowering drugs showed no significant association with mortality and adverse outcomes. Given the close relationship between diabetes and COVID-19 and the limited evidence on the role of glucose-lowering drugs, prospective studies are needed.

Keywords: Type 2 diabetes mellitus, Glucose-lowering drug, Coronavirus disease 2019

\section{Background}

The novel coronavirus disease 2019 (COVID-19) caused by the severe acute respiratory syndrome coronavirus 2 (SARS-CoV-2) initially emerged in China in December 2019 and quickly spread around the world, causing a global pandemic [1].

For patients with COVID-19, diabetes has been reported as one of the most frequent comorbidities, occurring in around $20 \%$ of patients $[2,3]$. It is well known that type 2 diabetes mellitus (T2DM) may negatively impact clinical outcomes in patients with COVID-19. Possible poor outcomes include moderate and severe cases of COVID-19 disease, a higher rate of hospitalized patients in intensive care unit (ICU), a higher rate of treatment with anti-interleukin 6 receptor antibody (tocilizumab), and higher mortality [4]. Furthermore, it has been reported that hyperglycemia during COVID-19 infection, and particularly at hospital admission, has been associated with worse COVID-19 outcomes and could be a prognostic factor for worse outcomes in patients with or without T2DM $[5,6]$. Indeed, patients with hyperglycemia may experience reduced effect of anti-COVID-19 therapies, particularly tocilizumab, an anti-interleukin 6 receptor antibody indicated for patients with moderate-to-severe COVID-19 pneumonia [7]. Thus, not only T2DM status but also the presence of hyperglycemia could have unfavorable effects on hospital admission, clinical outcomes, and drug therapy, leading to a worse prognosis in COVID-19 patients [5, 6].

However, to date, there is no conclusive evidence in regard to the potential implications on adverse outcomes of glucose-lowering drugs in patients with COVID-19 [8-11]. In a recent study, the use of oral glucose-lowering medications (metformin, $\alpha$-glucosidase, secretagogues, and dipeptidyl peptidase-4 inhibitors (DPP-4i)) showed neutral effects on in-hospital mortality and the composite outcome of poor prognosis defined as progression to severe or critical illness and in-hospital death. In contrast, insulin usage was associated with a greater risk of poor prognosis compared to not using it [12]. Other studies have reported a beneficial effect of metformin and DPP-4i on clinical outcomes in patients hospitalized with COVID-19 [13, 14]. The potential effects of these medications on COVID-19-related pathological mechanisms, such as systemic inflammation and endothelial dysfunction, have been proposed as the underlying mechanisms of possible benefits in reports on mechanistic hypotheses and preliminary data $[10,15,16]$.

We conducted this study based on the premise that patients with T2DM seem particularly prone to a worse prognosis if infected by SARS-CoV-2, and in response to the fact that there is only controversial, limited evidence on the role of glucose-lowering drugs on clinical adverse outcomes. Our main objective was to examine the association between in-hospital death and each routine athome glucose-lowering drug both individually and in combination with metformin in patients with T2DM admitted for COVID-19. We also evaluated their association with the composite outcome of need for ICU admission, invasive and non-invasive mechanical ventilation, or in-hospital death as well as on the development 
of in-hospital complications and a long-time hospital stay.

\section{Methods \\ Study design and population}

We selected all patients with T2DM included in the Spanish Society of Internal Medicine's registry of COVID-19 patients (SEMI-COVID-19 Registry) [17] from March 1, 2020, to the study's cutoff date of July 19, 2020. The SEMI-COVID-19 Registry is an ongoing, observational, multicenter, nationwide cohort of patients admitted for COVID-19 in Spain from March 1, 2020, whose main objective is to obtain detailed information on the epidemiology, clinical progress, and treatment received by patients with COVID-19 in real-world clinical practice at admission and during hospitalization. It retrospectively compiles sociodemographic variables, previous medical history, routine at-home treatments, clinical presentation, the patient's clinical condition (including the degree of functional dependence as evaluated by the Barthel Index [18] and the presence of comorbidities as evaluated by the Charlson Comorbidity Index [19]), laboratory test results (blood gas analysis, complete blood count, coagulation tests, glucose, creatinine, urea, lactate dehydrogenase, alanine aminotransferase, aspartate aminotransferase, alkaline phosphatase, bilirubin, sodium, potassium, triglycerides, creatine kinase, ferritin, lactic acid, c-reactive protein, procalcitonin, interleukin 6, d-dimer, troponin, albumin), radiological findings (chest X-ray, chest-computerized tomography scan, lung ultrasound), clinical management, hospital complications, hospital stay, and in-hospital death from the first admission for COVID-19 of patients who are aged 18 years of age or older.

An online electronic data capture system was developed for the registry, including a database manager along with procedures for the verification of data and contrasting of information against the original medical record in order to ensure the best possible quality of data collection. The database platform is hosted on a secure server. All information contained in the database, the configuration of the information within the database, and the database itself are fully encrypted. Every clientserver data transfer is encrypted through a valid TLS certificate. Daily backups are performed in order to ensure data integrity.

A diagnosis of T2DM was ascertained either through a T2DM diagnosis in medical records or a self-reported diagnosis confirmed by medical records reviewed by physicians. T2DM was defined according to the most recent American Diabetes Association guidelines [20].

In-hospital complications include the onset of at least one of the following: secondary bacterial pneumonia, acute respiratory distress syndrome, acute heart failure, arrhythmia, acute coronary syndrome, myocarditis, epileptic seizures, stroke, shock, sepsis, acute kidney failure, disseminated intravascular coagulation, venous thromboembolism, multiple organ dysfunction syndrome, acute limb ischemia, ICU admission, and need for ventilation support, including invasive and non-invasive mechanical ventilation or high-flow oxygen therapy. A long hospital stay was defined as hospitalization longer than the median length of stay in days for all patients included in this study.

\section{Diagnosis and severity grade of COVID-19 disease}

The presence of COVID-19 in respiratory specimens was established by RNA detection of SARS-CoV-2 using real-time reverse transcription-polymerase chain reaction methods as indicated by the international literature on COVID-19 infection [1]. Diagnostic testing for SARS-CoV-2 was performed in appropriately equipped laboratories by staff trained in the relevant technical and safety procedures. The COVID-19 pneumonia severity grade was established according to the patient's clinical condition: mild grade (symptoms without evidence of pneumonia or hypoxia), moderate grade (clinical signs of pneumonia but no signs of severe pneumonia, including basal oxygen saturation $\geq 92 \%$ ), severe grade (clinical signs of pneumonia plus one of the following: basal oxygen saturation $<92 \%$, resting respiratory rate $>30$ breaths/min, severe respiratory distress), and critical grade (sepsis or shock with acute respiratory distress syndrome and/or multiple organ dysfunction or failure).

\section{Study outcomes}

The primary outcome was in-hospital death according to each at-home glucose-lowering drug in monotherapy or in dual therapy with metformin. Secondary outcomes were the following: first, a composite outcome including the need for ICU admission, invasive and non-invasive mechanical ventilation, or in-hospital death; second, inhospital complications; and third, a long hospital stay.

\section{Statistical analysis}

The characteristics of patients with T2DM included in the registry were analyzed using descriptive statistics. Continuous and categorical variables were expressed as means \pm standard deviation and as absolute value and percentage, respectively. The differences between the groups were determined using the two-sample Student's $t$ test or the Mann-Whitney-Wilcoxon rank-sum test for continuous variables and Pearson's chi-squared test for categorical variables. Values were considered to be statistically significant when $p<0.05$.

We grouped patients according to glucose-lowering drugs in monotherapy and in combination with metformin. In order to match each patient in one of these 
groups with a patient of another group receiving other glucose-lowering drugs in a 1:1 manner, propensity scores using nearest neighbor matching with a caliper of 0.1 and a greedy matching algorithm were used. The probability of a patient being treated with one glucoselowering drug as opposed to other glucose-lowering drugs was estimated using a logistic regression model that included variables that could have affected the outcomes as independent variables (age; gender; history of smoking, hypertension; dyslipidemia; chronic kidney disease; cerebrovascular disease; chronic obstructive pulmonary disease; atrial fibrillation; coronary artery disease; heart failure; obesity; dementia; Barthel Index score; and Charlson Comorbidity Index score; treatment with angiotensin-converting enzyme inhibitor, angiotensin II receptor blocker, anticoagulant, and statin; admission blood glucose; serum creatinine; and transaminase levels). In order to assess the adequacy of propensity matching, we used the standardized mean difference found in patient characteristics after matching. There was considered to be a significant imbalance in the group if a standardized mean difference between baseline variables of greater than $10 \%$ was found.

According to the findings of Austin [21], the statistical models for paired samples are preferable to models for the analysis of independent samples. For this reason, in order to evaluate the association between treatment and study outcomes, both conditional logit and mixed effect (matched pairs as random effects) logistic regressions were used. In case the sample size was smaller than 100, the McNemar test for matched data was performed to evaluate the association. We also performed univariate and multivariate logistic regression models adjusted with confounding variables in order to estimate the treatment effect using the totality of the data, as a sensitivity analysis. Statistical analyses were performed using the $R$ software, version 3.6.2.

\section{Results}

Baseline clinical variables and treatments

A total of 2666 patients with T2DM admitted for COVID-19 were included in this study. Baseline sociodemographic, clinical, and therapeutic variables are shown in Table 1. In regard to glucose-lowering drugs, metformin was the most frequently used $(60.8 \%)$, followed by DPP-4i (30.2\%), insulin (27.6\%), sodium-glucose cotransporter 2 inhibitor (SGLT-2i) (11.3\%), and glucagon-like peptide-1 receptor agonist (GLP-1RA) (4.8\%).

Of the total number of patients, 1297 (48.6\%) were treated with glucose-lowering drugs in monotherapy (825 with metformin (63.6\%), 180 with DPP-4i (13.9\%), and 292 with insulin (22.5\%)). Dual therapy of metformin in combination with other glucose-lowering drugs was used in 465 patients (17.4\%) (288 with metformin plus DPP-4i (61.9\%), 67 metformin plus SGLT-2i (14.4\%), and 110 metformin plus insulin (23.7\%)). Following 1:1 propensity score matching of each glucoselowering drug alone or in combination with metformin versus the other glucose-lowering drugs, 249 patients were included in the metformin group, 105 in the DPP$4 \mathrm{i}$ group, 129 in the insulin group, 127 in the metformin plus DPP-4i group, 34 in the metformin plus SGLT-2i group, and 67 in the metformin plus insulin group. No patients were identified with GLP-1RA alone, SGLT-2i alone, or metformin plus GLP-1RA. A flow chart for pre- and post-propensity score matching showing patients in each compared group can be found in Fig. 1.

\section{Pre- and post-propensity matching characteristics}

The pre- and post-propensity score matching of the baseline sociodemographic and clinical characteristics of each glucose-lowering drug alone or in combination with metformin versus the other glucose-lowering drugs used by patients before hospitalization for COVID-19 are shown in Additional file 1: Table S1; Additional file 2: Table S2; Additional file 3: Table S3; Additional file 4: Table S4; Additional file 5: Table S5; and Additional file 6: Table S6. After propensity matching, the groups were well-balanced, and only negligible differences were observed (standardized mean difference $\leq 0.1$ ).

\section{Associations between treatments and study outcomes}

After propensity score matching, no differences were found in in-hospital deaths; the composite outcome of need for ICU admission, invasive and non-invasive mechanical ventilation, or in-hospital death; in-hospital complications; and a long-time hospital stay according to the glucose-lowering drug used. None of the at-home glucose-lowering drugs analyzed showed significant association with study outcomes after applying both the conditional logit and mixed effect logistic regression if the sample size was $\geq 100$ or McNemar test if the sample size was $<100$. The association between each glucoselowering drug group and the study outcomes after propensity matching is shown in Tables 2 and 3.

Before matching, patients treated at home with metformin in monotherapy, compared to patients who were treated with other glucose-lowering drugs, had a lower rate of in-hospital deaths $(29.6 \%$ vs $36.8 \%, p=0.005)$; the composite outcome of ICU admission, mechanical ventilation, or in-hospital death ( $35.8 \%$ vs $41.9 \%, p=0.023$ ); and in-hospital complications $(52.7 \%$ vs $59.4 \%, p=$ 0.013). A lower in-hospital death rate was also found in patients with metformin plus DPP-4i $(27.8 \%$ vs $35.4 \%$, $p=0.044$ ) and in patients with metformin plus SGLT-2i $(19.4 \%$ vs $39.1 \%, p=0.003)$. On the other hand, a higher rate of in-hospital deaths was observed among patients with DPP-4i alone and insulin alone $(41.7 \%$ vs $31.2 \%$, 
Table 1 Baseline sociodemographic clinical and therapeutic characteristics of patients with type 2 diabetes mellitus hospitalized for coronavirus disease 2019

\begin{tabular}{|c|c|}
\hline Variables & $n=2666$ \\
\hline Age (years) & $74.9 \pm 8.4$ \\
\hline Male gender & $1647(61.9 \%)$ \\
\hline Obesity & $766(31.6 \%)$ \\
\hline Admission blood glucose (mg/dL) & $153 \pm 45.7$ \\
\hline Admission serum creatinine (mg/dL) & $1.03 \pm 0.23$ \\
\hline Admission aspartate aminotransferase $(U / L)$ & $31 \pm 8$ \\
\hline Admission alanine aminotransferase (U/L) & $26 \pm 6$ \\
\hline Metformin & $1618(60.8 \%)$ \\
\hline DPP-4i & $791(30.2 \%)$ \\
\hline GLP1-1RA & $127(4.8 \%)$ \\
\hline SGLT-2i & $296(11.3 \%)$ \\
\hline Insulin & $723(27.6 \%)$ \\
\hline Angiotensin-converting enzyme inhibitor & $724(27.4 \%)$ \\
\hline Angiotensin I| receptor blocker & $789(29.9 \%)$ \\
\hline Statin & $1534(58.0 \%)$ \\
\hline Antiplatelet & $643(24.8 \%)$ \\
\hline Anticoagulant & $(16.3 \%)$ \\
\hline History of smoking & 955 (35.8\%) \\
\hline Hypertension & $2026(76.2 \%)$ \\
\hline Dyslipidemia & $1730(65.0 \%)$ \\
\hline Chronic kidney disease & $352(13.2 \%)$ \\
\hline Atrial fibrillation & $444(16.7 \%)$ \\
\hline Coronary artery disease & $610(22.9 \%)$ \\
\hline Heart failure & $445(16.7 \%)$ \\
\hline Chronic obstructive pulmonary disease & $346(13.0 \%)$ \\
\hline Liver disease & $172(6.5 \%)$ \\
\hline Cancer & $353(13.3 \%)$ \\
\hline Stroke & $322(12.1 \%)$ \\
\hline Dementia & $384(14.4 \%)$ \\
\hline Depression & $308(11.6 \%)$ \\
\hline Moderate-severe functional dependence & $636(24.2 \%)$ \\
\hline Moderate-severe comorbidity & $2338(90.7 \%)$ \\
\hline \multicolumn{2}{|l|}{ Disease severity } \\
\hline Moderate & $1891(70.9 \%)$ \\
\hline Severe & $714(26.8 \%)$ \\
\hline Critical & $61(2.3 \%)$ \\
\hline Hydroxychloroquine & $2185(82.0 \%)$ \\
\hline Chloroquine & $72(2.7 \%)$ \\
\hline Lopinavir/ritonavir & $1195(44.8 \%)$ \\
\hline Azithromycin & $1595(59.8 \%)$ \\
\hline Remdesivir & $36(1.4 \%)$ \\
\hline Interferon- $\beta$ & $276(10.4 \%)$ \\
\hline
\end{tabular}

Table 1 Baseline sociodemographic clinical and therapeutic characteristics of patients with type 2 diabetes mellitus hospitalized for coronavirus disease 2019 (Continued)

\begin{tabular}{ll}
\hline Variables & $\boldsymbol{n}=\mathbf{2 6 6 6}$ \\
\hline Corticosteroids & $1186(44.5 \%)$ \\
Tocilizumab & $215(8.1 \%)$ \\
\hline
\end{tabular}

Values are shown as mean \pm standard deviations, absolute values, and percentages

The degree of functional dependence was assessed using the Barthel Index. The presence of comorbidities was assessed using the Charlson

Comorbidity Index

DPP-4i dipeptidyl peptidase-4 inhibitors, GLP-1RA glucagon-like peptide-1 receptor agonist, $\mathrm{mg} / \mathrm{dL}$ milligram/deciliter, $S G L T$-2i sodium-glucose cotransporter 2 inhibitor, $U / L$ unit/liter

$p=0.007 ; 38 \%$ vs $29.6 \%, p=0.007$, respectively). Patients with insulin also had a higher rate of the composite outcome of the need for ICU admission, mechanical ventilation, or in-hospital death $(42.8 \%$ vs $36.2 \%, p=0.039)$. Patients treated with metformin and insulin were found to have higher rates of in-hospital complications $(66.4 \%$ vs $55.0 \%, p=0.030$ ).

On the univariate models, metformin was significantly associated with a lower rate of in-hospital deaths (odds ratio (OR) $0.73,95 \%$ confidence interval (CI) 0.58-0.90, $p=0.004)$; the need for ICU admission, mechanical ventilation, or in-hospital death (OR 0.78 , 95\% CI $0.63-$ $0.96, p=0.020$ ); and in-hospital complications (OR 0.76, 95\% CI 0.62-0.94, $p=0.011$ ). Metformin in combination with DPP-4i was associated with a lower in-hospital death rate (OR 0.70, 95\% CI 0.50-0.98, $p=0.036$ ). Otherwise, DPP-4i and insulin were associated with higher in-hospital deaths (OR 1.56, 95\% CI 1.13-2.14, $p=0.006$; OR $1.45,95 \%$ CI 1.11-1.88, $p=0.006$; respectively). The composite outcome of the need for ICU admission, mechanical ventilation, or in-hospital death was also higher in patients on insulin (OR 1.32, 95\% CI $1.02-1.71, p=0.033)$. These associations were not found when models were fully adjusted using multivariate logistic regression. The association between each glucoselowering drug group and the study outcomes before propensity score matching is shown in Tables 4 and 5 .

\section{Discussion}

Our study found that none of the at-home glucoselowering drugs analyzed (metformin, DPP-4i, insulin, metformin plus DPP-4i, metformin plus SGLT-2i, and metformin plus insulin) showed a significant association with in-hospital deaths; the composite outcome of the need for ICU, mechanical ventilation, or in-hospital death; in-hospital complications; or a long-time hospital stay.

Currently, only limited evidence is available on the role of glucose-lowering drugs in adverse clinical outcomes. Recently, a study conducted by Chen et al. [12] focused on the impact of glucose-lowering medications 


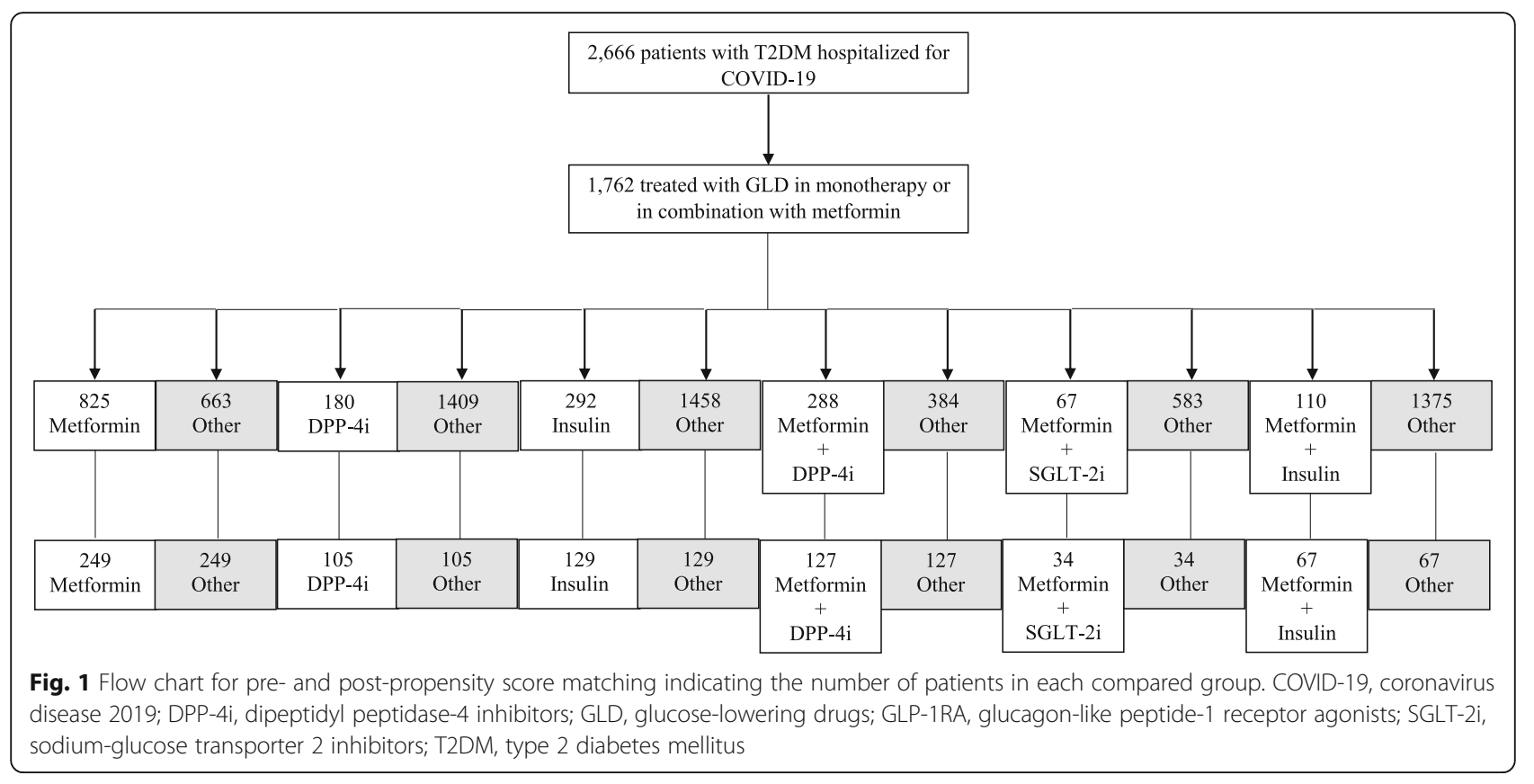

on the clinical outcomes of patients with diabetes and COVID-19, showing that insulin users had a greater risk of a composite outcome of progression to severe or critical illness and in-hospital death compared with noninsulin users (OR 3.58, 95\% CI 1.37-9.35, $p=0.009$ ). However, none of the glucose-lowering medications (metformin, DPP-4i, $\alpha$-glucosidase, secretagogues, and insulin) was associated with in-hospital deaths. In our study, all glucose-lowering medications evaluated, including oral drugs and insulin, had no significant association with in-hospital adverse outcomes in patients admitted for COVID-19. The differences in the definitions of the outcomes of clinical complications during the hospitalization, the methodology used, and the sample size could explain the difference found regarding the role of insulin. We included an important number of patients taking glucose-lowering drugs and performed a propensity score matching that included variables that could affect the treatment choice or adverse outcomes as independent variables.

Two observational studies have specifically evaluated the role of metformin and DPP-4i in patients admitted for COVID-19 [13, 14]. The study focused on metformin, conducted by Bramante et al. [13] in the USA, found no significant reduction in in-hospital mortality in the overall sample, which is in line with our results. However, when a subgroup analysis was performed, women with obesity and T2DM who used metformin were observed to have a significantly lower mortality rate. Metformin has been proposed to reduce levels of IL-10, IL-6, and TNF $\alpha$-important mediators in macrophage activation and cytokine release; improves neutrophil/ lymphocyte ratio; stabilizes mast cells; decreases thrombosis; and improves endothelial function, thus reducing the adverse impacts of mortality and complication in patients with COVID-19 [13, 22-27]. In the study of Rhee et al. [14] from Korea, protective effects against severe/lethal cases were shown in patients with T2DM who used DPP-4i in monotherapy or in combination with renin-angiotensin system blockers after adjusting for age, gender, comorbidity, and medications [14]. It is known that DPP-4i have anti-fibrotic activity and modulate inflammation and that these properties could be helpful in reducing the progression towards a hyperinflammatory state associated with severe COVID-19 [14, 28, 29]. All these major inflammatory mechanisms observed in some COVID-19 patients suggest that the endothelium could be a key target organ in COVID-19 infection [30]. The role of renin-angiotensin system blockers in hypertensive patients with COVID-19 has also been widely discussed [31]. Although hypertension has been associated with a higher risk of mortality, recent studies have shown that previous treatment with angiotensinconverting enzyme inhibitors and angiotensin II receptor blockers did not alter the outcomes in hypertensive patients [31, 32]. In our study, no significant associations with adverse clinical outcomes were found among users of DPP-4i and users of the other glucose-lowering drugs. This difference could be explained by the different methodology used in our study, which includes an important adjustment using a propensity score matching in order to compare the two groups of glucose-lowering drugs, as well as the differences in the baseline characteristics, with older patients and higher comorbidity found in 
Table 2 Association between metformin, dipeptidyl peptidase-4 inhibitors, insulin, and metformin plus dipeptidyl peptidase-4 inhibitors and study outcomes after propensity score matching

\begin{tabular}{|c|c|c|c|c|c|c|c|}
\hline \multirow[t]{2}{*}{ Outcomes } & \multirow[t]{2}{*}{ Treatment groups } & & & \multicolumn{2}{|c|}{$\begin{array}{l}\text { Conditional logit logistic } \\
\text { regression }\end{array}$} & \multicolumn{2}{|c|}{$\begin{array}{l}\text { Mixed effect logistic } \\
\text { regression }\end{array}$} \\
\hline & & & & OR $(95 \% \mathrm{Cl})$ & $p$ value & OR $(95 \% \mathrm{Cl})$ & $p$ value \\
\hline & $\begin{array}{l}\text { Metformin } \\
(n=249)\end{array}$ & $\begin{array}{l}\text { Other GLD } \\
(n=249)\end{array}$ & $p$ value & & & & \\
\hline In-hospital deaths & $79(31.7 \%)$ & $79(31.7 \%)$ & 1.000 & $1.15(0.78-1.71)$ & 0.482 & $1.16(0.78-1.72)$ & 0.482 \\
\hline $\begin{array}{l}\text { ICU admission, mechanical ventilation, } \\
\text { or in-hospital death }\end{array}$ & 91 (36.5\%) & $88(35.4 \%)$ & 0.852 & $1.05(0.73-1.51)$ & 0.780 & $1.05(0.73-1.52)$ & 0.779 \\
\hline In-hospital complications & $146(58.6 \%)$ & $139(55.8 \%)$ & 0.587 & $1.18(0.81-1.72)$ & 0.392 & $1.17(0.81-1.70)$ & 0.398 \\
\hline \multirow[t]{2}{*}{ Long-time hospital stay } & $59(23.7 \%)$ & $45(18.1 \%)$ & 0.152 & $1.49(0.95-2.31)$ & 0.079 & $1.49(0.96-2.33)$ & 0.075 \\
\hline & DPP-4i $(n=105)$ & $\begin{array}{l}\text { Other GLD } \\
(n=105)\end{array}$ & $p$ value & & & & \\
\hline In-hospital deaths & 41 (39.0\%) & $44(41.9 \%)$ & 0.779 & $1.05(0.66-2.13)$ & 0.521 & $1.05(0.67-2.11)$ & 0.562 \\
\hline $\begin{array}{l}\text { ICU admission, mechanical ventilation, } \\
\text { or in-hospital death }\end{array}$ & 45 (42.9\%) & $42(40.0 \%)$ & 0.780 & $1.12(0.65-1.92)$ & 0.680 & $1.12(0.65-1.95)$ & 0.675 \\
\hline In-hospital complications & $66(62.9 \%)$ & $70(66.7 \%)$ & 0.665 & $0.94(0.67-2.12)$ & 0.480 & $0.94(0.67-2.13)$ & 0.481 \\
\hline \multirow[t]{2}{*}{ Long-time hospital stay } & $23(21.9 \%)$ & $24(22.9 \%)$ & 1.000 & $0.83(0.38-1.46)$ & 0.400 & $0.84(0.39-1.46)$ & 0.406 \\
\hline & Insulin $(n=129)$ & $\begin{array}{l}\text { Other GLD } \\
(n=129)\end{array}$ & $p$ value & & & & \\
\hline In-hospital deaths & $51(39.5 \%)$ & $46(35.7 \%)$ & 0.607 & $1.15(0.65-1.97)$ & 0.590 & $1.15(0.65-1.97)$ & 0.598 \\
\hline $\begin{array}{l}\text { ICU admission, mechanical ventilation, } \\
\text { or in-hospital death }\end{array}$ & $57(44.2 \%)$ & $54(41.9 \%)$ & 0.802 & $1.10(0.67-1.80)$ & 0.710 & $1.10(0.67-1.81)$ & 0.706 \\
\hline In-hospital complications & $49(38.9 \%)$ & $46(35.7 \%)$ & 0.796 & $1.00(0.58-1.67)$ & 0.930 & $1.00(0.57-1.67)$ & 0.912 \\
\hline \multirow[t]{2}{*}{ Long-time hospital stay } & $22(17.1 \%)$ & $34(26.4 \%)$ & 0.097 & $0.63(0.37-1.20)$ & 0.158 & $0.63(0.37-1.21)$ & 0.159 \\
\hline & $\begin{array}{l}\text { Metformin + DPP- } 4 \mathrm{i} \\
(n=127)\end{array}$ & $\begin{array}{l}\text { Other GLD } \\
(n=127)\end{array}$ & $p$ value & & & & \\
\hline In-hospital deaths & $29(22.8 \%)$ & 37 (29.1\%) & 0.317 & $0.73(0.40-1.28)$ & 0.270 & $0.72(0.39-1.27)$ & 0.251 \\
\hline $\begin{array}{l}\text { ICU admission, mechanical ventilation, } \\
\text { or in-hospital death }\end{array}$ & $40(31.5 \%)$ & $45(35.4 \%)$ & 0.592 & $0.84(0.50-1.41)$ & 0.510 & $0.84(0.49-1.41)$ & 0.503 \\
\hline In-hospital complications & $67(52.8 \%)$ & $70(55.1 \%)$ & 0.801 & $0.87(0.51-1.46)$ & 0.596 & $0.86(0.50-1.47)$ & 0.592 \\
\hline Long-time hospital stay & $29(22.8 \%)$ & $26(20.5 \%)$ & 0.761 & $1.17(0.62-2.23)$ & 0.627 & $1.17(0.62-2.19)$ & 0.632 \\
\hline
\end{tabular}

Data are shown as absolute values and percentages. A significant imbalance in the group was considered if a standardized mean difference between baseline variables of greater than $10 \%$ was found. Values were considered to be statistically significant when $p<0.05$

DPP-4i dipeptidyl peptidase-4 inhibitors, GLD glucose-lowering drugs, ICU intensive care unit, OR odds ratio, $95 \% \mathrm{CI} 95 \%$ confidence interval

Table 3 Association between metformin plus sodium-glucose transporter 2 inhibitors and metformin plus insulin and study outcomes after propensity score matching

\begin{tabular}{|c|c|c|c|c|}
\hline \multirow[t]{2}{*}{ Outcomes } & \multicolumn{3}{|l|}{ Treatment groups } & \multirow[t]{2}{*}{ McNemar test } \\
\hline & Metformin + SGLT-2i $(n=34)$ & Other GLD $(n=34)$ & $p$ value & \\
\hline In-hospital deaths & $6(17.6 \%)$ & $10(29.4 \%)$ & 0.391 & 1.000 \\
\hline ICU admission, mechanical ventilation, or in-hospital death & $10(29.4 \%)$ & $11(32.4 \%)$ & 0.987 & 0.965 \\
\hline In-hospital complications & $19(55.9 \%)$ & $22(64.7 \%)$ & 0.620 & 0.201 \\
\hline \multirow[t]{2}{*}{ Long-time hospital stay } & $6(17.6 \%)$ & $4(11.8 \%)$ & 0.732 & 0.838 \\
\hline & Metformin + insulin $(n=67)$ & Other GLD $(n=67)$ & $p$ value & \\
\hline In-hospital deaths & $24(35.8 \%)$ & $23(34.3 \%)$ & 1.000 & 0.521 \\
\hline ICU admission, mechanical ventilation, or in-hospital death & $46(68.7 \%)$ & $36(53.7 \%)$ & 0.112 & 0.111 \\
\hline In-hospital complications & $44(65.7 \%)$ & $36(53.7 \%)$ & 0.218 & 0.480 \\
\hline Long-time hospital stay & $15(22.4 \%)$ & $13(19.4 \%)$ & 0.832 & 0.870 \\
\hline
\end{tabular}

Data are shown as absolute values and percentages. Values were considered to be statistically significant when $p<0.05$ GLD glucose-lowering drugs, ICU intensive care unit, SGLT-2i sodium-glucose transporter 2 inhibitors 
Table 4 Association between metformin, dipeptidyl peptidase-4 inhibitors, insulin, and metformin plus dipeptidyl peptidase-4 inhibitors and study outcomes before propensity score matching

\begin{tabular}{|c|c|c|c|c|c|c|c|}
\hline \multirow[t]{2}{*}{ Outcomes } & \multirow[t]{2}{*}{ Treatment groups } & & & \multicolumn{2}{|c|}{ Univariate model } & \multicolumn{2}{|c|}{ Multivariate model } \\
\hline & & & & OR $(95 \% \mathrm{CI})$ & $p$ value & OR $(95 \% \mathrm{Cl})$ & $p$ value \\
\hline & Metformin $(n=825)$ & $\begin{array}{l}\text { Other GLD } \\
(n=663)\end{array}$ & $p$ value & & & & \\
\hline In-hospital deaths & $244(29.6 \%)$ & $244(36.8 \%)$ & 0.005 & $0.73(0.58-0.90)$ & 0.004 & $1.10(0.76-1.60)$ & 0.616 \\
\hline $\begin{array}{l}\text { ICU admission, mechanical ventilation, } \\
\text { or in-hospital death }\end{array}$ & $295(35.8 \%)$ & $278(41.9 \%)$ & 0.023 & $0.78(0.63-0.96)$ & 0.020 & $1.03(0.73-1.44)$ & 0.883 \\
\hline In-hospital complications & $435(52.7 \%)$ & $394(59.4 \%)$ & 0.013 & $0.76(0.62-0.94)$ & 0.011 & $1.08(0.77-1.50)$ & 0.666 \\
\hline \multirow[t]{2}{*}{ Long-time hospital stay } & $195(23.6 \%)$ & $149(22.5 \%)$ & 0.584 & $1.08(0.85-1.38)$ & 0.542 & $1.41(0.96-2.09)$ & 0.080 \\
\hline & DPP-4i $(n=180)$ & $\begin{array}{l}\text { Other GLD } \\
(n=1409)\end{array}$ & $p$ value & & & & \\
\hline In-hospital deaths & 75 (41.7\%) & $440(31.2 \%)$ & 0.007 & $1.56(1.13-2.14)$ & 0.006 & $1.39(0.64-1.67)$ & 0.876 \\
\hline $\begin{array}{l}\text { ICU admission, mechanical ventilation, } \\
\text { or in-hospital death }\end{array}$ & $80(44.4 \%)$ & $531(37.7 \%)$ & 0.119 & $1.30(0.95-1.78)$ & 0.102 & $0.97(0.61-1.52)$ & 0.890 \\
\hline In-hospital complications & $113(62.8 \%)$ & $775(55.0 \%)$ & 0.104 & $1.33(0.96-1.84)$ & 0.089 & $0.95(0.59-1.54)$ & 0.842 \\
\hline \multirow[t]{2}{*}{ Long-time hospital stay } & 35 (19.4\%) & $321(22.8 \%)$ & 0.333 & $0.81(0.54-1.18)$ & 0.288 & $0.90(0.52-1.52)$ & 0.703 \\
\hline & Insulin ( $n=292)$ & Other GLD $(n=1458)$ & $p$ value & & & & \\
\hline In-hospital deaths & $111(38.0 \%)$ & $431(29.6 \%)$ & 0.007 & $1.45(1.11-1.88)$ & 0.006 & $1.11(0.70-1.75)$ & 0.652 \\
\hline $\begin{array}{l}\text { ICU admission, mechanical ventilation, } \\
\text { or in-hospital death }\end{array}$ & $125(42.8 \%)$ & $528(36.2 \%)$ & 0.039 & $1.32(1.02-1.71)$ & 0.033 & $1.09(0.71-1.66)$ & 0.699 \\
\hline In-hospital complications & $170(58.2 \%)$ & $786(53.9 \%)$ & 0.143 & $1.23(0.95-1.60)$ & 0.126 & $0.96(0.62-1.47)$ & 0.834 \\
\hline \multirow[t]{2}{*}{ Long-time hospital stay } & $74(25.3 \%)$ & $327(22.4 \%)$ & 0.357 & $1.16(0.86-1.55)$ & 0.318 & $0.699(0.43-1.12)$ & 0.145 \\
\hline & $\begin{array}{l}\text { Metformin + DPP- } 4 \mathrm{i} \\
(n=288)\end{array}$ & $\begin{array}{l}\text { Other GLD } \\
(n=384)\end{array}$ & $p$ value & & & & \\
\hline In-hospital deaths & $80(27.8 \%)$ & $136(35.4 \%)$ & 0.044 & $0.70(0.50-0.98)$ & 0.036 & $0.71(0.40-1.28)$ & 0.257 \\
\hline $\begin{array}{l}\text { ICU admission, mechanical ventilation, } \\
\text { or in-hospital death }\end{array}$ & $104(36.1 \%)$ & $159(41.4 \%)$ & 0.159 & $0.79(0.57-1.08)$ & 0.137 & $0.82(0.50-1.36)$ & 0.452 \\
\hline In-hospital complications & $160(55.6 \%)$ & $226(58.9 \%)$ & 0.304 & $0.84(0.61-1.15)$ & 0.268 & $1.01(0.63-1.62)$ & 0.975 \\
\hline Long-time hospital stay & $71(24.7 \%)$ & $95(24.7 \%)$ & 1.000 & $1.00(0.71-1.44)$ & 0.963 & $1.42(0.82-2.47)$ & 0.217 \\
\hline
\end{tabular}

Data are shown as absolute values and percentages. Values were considered to be statistically significant when $p<0.05$ $D P P-4 i$ dipeptidyl peptidase-4 inhibitors, GLD glucose-lowering drugs, ICU intensive care unit, OR odds ratio, $95 \% \mathrm{Cl} 95 \%$ confidence interval

Table 5 Association between metformin plus sodium-glucose transporter 2 inhibitors and metformin plus insulin and study outcomes before propensity score matching

\begin{tabular}{|c|c|c|c|}
\hline \multirow[t]{2}{*}{ Outcomes } & \multicolumn{3}{|l|}{ Treatment groups } \\
\hline & Metformin + SGLT-2i $(n=67)$ & Other GLD $(n=583)$ & $p$ value \\
\hline In-hospital deaths & $13(19.4 \%)$ & $228(39.1 \%)$ & 0.003 \\
\hline ICU admission, mechanical ventilation, or in-hospital death & $35(52.2 \%)$ & 375 (64.3\%) & 0.051 \\
\hline In-hospital complications & $33(49.3 \%)$ & $351(60.2 \%)$ & 0.132 \\
\hline \multirow[t]{2}{*}{ Long-time hospital stay } & $8(11.9 \%)$ & $130(22.3 \%)$ & 0.087 \\
\hline & Metformin + insulin $(n=110)$ & Other GLD $(n=1375)$ & $p$ value \\
\hline In-hospital deaths & $43(39.1 \%)$ & 445 (32.4\%) & 0.175 \\
\hline ICU admission, mechanical ventilation, or in-hospital death & $76(69.1 \%)$ & $810(58.9 \%)$ & 0.084 \\
\hline In-hospital complications & $73(66.4 \%)$ & $756(55.0 \%)$ & 0.030 \\
\hline Long-time hospital stay & $21(19.1 \%)$ & $320(23.3 \%)$ & 0.360 \\
\hline
\end{tabular}

Data are shown as absolute values and percentages. Values were considered to be statistically significant when $p<0.05$ GLD glucose-lowering drugs, ICU intensive care unit, SGLT-2i sodium-glucose transporter 2 inhibitors 
our study compared with Rhee et al.'s study (mean age of $74.9 \pm 8.4$ vs $63.7 \pm 12.2$ years old).

Other glucose-lowering treatments in our study, such as metformin in combination with SGLT-2i or with insulin, showed no significant differences compared with the other glucose-lowering treatments. No propensity score matching was performed for these two comparisons due to the small number of patients in these treatment groups, so no solid conclusions may be drawn. To our knowledge, no evidence exists about the role of SGLT-2i on adverse outcomes in patients with COVID-19. A hypothetical antiviral effect of SGLT-2i has been suggested, as these agents can increase lactate concentrations and decrease intracellular $\mathrm{pH}$, which could reduce the viral load [33].

Our findings are important because they provide valuable information on the role of at-home glucose-lowering drugs on adverse outcomes in patients with T2DM admitted for COVID-19. In addition, this is the first study to report the role of each glucose-lowering drugs alone or in combination with metformin compared to the other glucose-lowering drugs after a robust adjustment using a wide number of confounding variables. Furthermore, data were collected in a large multicenter, nationwide study. Nevertheless, these results should be considered within the context of several potential limitations. Despite the propensity matching analysis performed and due to the fact that the data were obtained retrospectively via medical records from the electronic medical record system, the possible effects of unmeasured confounding factors cannot be excluded. In addition, some glucose-lowering drug group comparisons had a small number of patients after propensity score matching that could be an explanation for the lack of significant differences. No other intermediate adverse outcomes were explored due to their reduced number. Furthermore, we did not record the characteristics of T2DM, such as glycemic control before hospitalization, duration of diabetes, blood glucose levels during the hospitalization, or in-hospital anti-hyperglycemic management. Lastly, the data provided about at-home glucose-lowering drugs did not include information on treatment adherence or treatment duration.

\section{Conclusions}

Our study found that the at-home use of metformin, DPP$4 \mathrm{i}$, insulin, metformin plus DPP-4i, metformin plus SGLT$2 \mathrm{i}$, and metformin plus insulin showed no significant association with in-hospital deaths; the composite outcome of need for ICU admission, mechanical ventilation, or inhospital death; in-hospital complications; or long-time hospital stay in patients with T2DM admitted for COVID-19. Given the large number of patients with T2DM and COVID-19 infection, the ominous relationship between these pathologies, and the limited evidence on the role of glucose-lowering drugs, prospective studies are needed.

\section{Supplementary Information}

Supplementary information accompanies this paper at https://doi.org/10. 1186/s12916-020-01832-2.

Additional file 1: Table S1. Pre- and post-propensity score matching of baseline sociodemographic and clinical characteristics of patients with type 2 diabetes mellitus admitted for coronavirus disease 2019 treated with metformin versus other glucose-lowering drugs.

Additional file 2: Table S2. Pre- and post-propensity score matching of baseline sociodemographic and clinical characteristics of patients with type 2 diabetes mellitus admitted for coronavirus disease 2019 treated with dipeptidyl peptidase-4 inhibitors versus other glucose-lowering drugs.

Additional file 3: Table S3. Pre- and post-propensity score matching of baseline sociodemographic and clinical characteristics of patients with type 2 diabetes mellitus admitted for coronavirus disease 2019 treated with insulin versus other glucose-lowering drugs.

Additional file 4: Table S4. Pre- and post-propensity score matching of baseline sociodemographic and clinical characteristics of patients with type 2 diabetes mellitus admitted for coronavirus disease 2019 treated with metformin plus dipeptidyl peptidase-4 inhibitors versus other glucose-lowering drugs.

Additional file 5: Table S5. Pre- and post-propensity score matching of baseline sociodemographic and clinical characteristics of patients with type 2 diabetes mellitus admitted for coronavirus disease 2019 treated with metformin plus sodium-glucose transporter 2 inhibitors versus other glucose-lowering drugs.

Additional file 6: Table S6. Pre- and post-propensity score matching of baseline sociodemographic and clinical characteristics of patients with type 2 diabetes mellitus admitted for coronavirus disease 2019 treated with metformin plus insulin versus other glucose-lowering drugs.

\section{Additional file 7.}

\section{Abbreviations}

Cl: Confidence interval; COVID-19: Coronavirus disease 2019; DPP4i: Dipeptidyl peptidase-4 inhibitors; GLP-1RA: Glucagon-like peptide-1 receptor agonist; ICU: Intensive care unit; OR: Odds ratio; SARS-Cov-2: Severe acute respiratory syndrome coronavirus 2; SGLT-2i: Sodium-glucose cotransporter 2 inhibitor; T2DM: Type 2 diabetes mellitus

\section{Acknowledgements}

We thank Ipek Guler Caamaño for her support to the statistical analysis, Claire Alexandra Conrad for her help with the final English language version, and the SEMI-COVID-19 Registry Coordinating Center, S\&H Medical Science Service, for their quality control data and logistic and administrative support.

\section{Authors' contributions}

LMPB contributed to the conception; design of the work; acquisition; interpretation of the data; writing - original draft preparation; writing - review and editing; and supervision. JDTP, MDLC, MMAG, FFJ, LJH, JAM, MRR, MM, MGG, BVM, JFS, JE, RGF, CMP, CJR, JJNL, IEAA, SPC, SFC, FAM, $B C R$, and JMRR made contributions to the acquisition of the data and revised the work. PPM contributed to the interpretation of the data; writing-review and editing; and supervision. RGH was a major contributor in the interpretation of the data; writing-original draft preparation; writing - review and editing; and supervision. All authors read and approved the final manuscript.

\section{Funding}

This research received no specific grant from any funding agency in the public, commercial, or not-for-profit sectors.

\section{Availability of data and materials}

All data generated or analyzed during this study are included in this published article and its supplementary information files. 


\section{Ethics approval and consent to participate}

Only patients who had previously given consent for their medical records to be used for medical research were included in this registry. Data confidentiality and patient anonymity were maintained at all times, in accordance with Spanish regulations on observational studies. Patientidentifying information was deleted before the database was analyzed. It is not possible to identify patients on an individual level either in this article or in the database. This study was also carried out in accordance with the Declaration of Helsinki and was approved by the Institutional Research Ethics Committee of Málaga on March 27, 2020 (Ethics Committee code: SEMICOVID-19 27-03-20), as per the guidelines of the Spanish Agency of Medicines and Medical Products.

\section{Consent for publication}

Not applicable.

\section{Competing interests}

The authors declare that they have no competing interests.

\section{Author details}

'Internal Medicine Department, Regional University Hospital of Málaga, Biomedical Research Institute of Málaga (IBIMA), University of Málaga (UMA), Avenida de Carlos Haya, s/n, 29010 Málaga, Spain. ${ }^{2}$ Lipids and Atherosclerosis Unit, Department of Internal Medicine, Maimonides Biomedical Research Institute of Cordoba (IMIBIC), Reina Sofia University Hospital, University of Cordoba, Córdoba, Spain. ${ }^{3} \mathrm{C}$ IBER Fisiopatología de la Obesidad y Nutrición (CIBEROBN), Instituto de Salud Carlos III, Madrid, Spain. ${ }^{4}$ Internal Medicine Department, 12 de Octubre University Hospital, Madrid, Spain. ${ }^{5}$ Internal Medicine Department, Gregorio Marañon University Hospital, Madrid, Spain. ${ }^{6}$ Internal Medicine Department, Bellvitge University Hospital, L'Hospitalet de Llobregat, Barcelona, Spain. ${ }^{7}$ Internal Medicine Department, Dr. Peset University Hospital, Valencia, Spain. ${ }^{8}$ Internal Medicine Department, San Juan de la Cruz Hospital, Úbeda (Jaén), Spain. ${ }^{9}$ Internal Medicine Department, León University Hospital Complex, León, Spain. ${ }^{10}$ Internal Medicine Department, Clinic Barcelona Hospital, Barcelona, Spain. ${ }^{11}$ Internal Medicine Department, Marina Baixa Hospital, Villajoyosa (Alicante), Spain. ${ }^{12}$ Internal Medicine Department, Tajo Hospital, Aranjuez (Madrid), Spain. ${ }^{13}$ Internal Medicine Department, Ferrol University Hospital Complex, Ferrol (A Coruña), Spain. ${ }^{14}$ Internal Medicine Department, Insular de Gran Canaria Hospital, Las Palmas de Gran Canaria, Spain. ${ }^{15}$ Internal Medicine Department, Marqués de Valdecilla University Hospital, Santander, Spain. ${ }^{16}$ Internal Medicine Department, Torrecárdenas Hospital, Almería, Spain. ${ }^{17}$ Internal Medicine Department, Severo Ochoa University Hospital, Leganés (Madrid), Spain. ${ }^{18}$ Internal Medicine Department, Valle del Nalón Hospital, Riaño (Langreo, Asturias), Spain. ${ }^{19}$ Internal Medicine Department, Vinalopó University Hospital, Elche (Alicante), Spain. ${ }^{20}$ Internal Medicine Department, Alto Guadalquivir Hospital, Andújar (Jaén), Spain. ${ }^{21}$ Department of Clinical Medicine, Miguel Hernandez University of Elche, Alicante, Spain.

\section{Received: 31 August 2020 Accepted: 29 October 2020}

\section{Published online: 16 November 2020}

\section{References}

1. Zhu N, Zhang D, Wang W, Li X, Yang B, Song J, et al. A novel coronavirus from patients with pneumonia in China, 2019. N Engl J Med. 2020;382:727-33.

2. Zhou F, Yu T, Du R, Fan G, Liu Y, Liu Z, et al. Clinical course and risk factors for mortality of adult inpatients with COVID-19 in Wuhan, China: a retrospective cohort study. Lancet. 2020;395:2054-62.

3. Remuzzi A, Remuzzi G. COVID-19 and Italy: what next? Lancet. 2020;395:1225-8.

4. Sardu C, D’Onofrio N, Balestrieri ML, Barbieri M, Rizzo R, Messina V, et al. Outcomes in patients with hyperglycemia affected by COVID-19: can we do more on glycemic control? Diabetes Care. 2020;43:1408-15.

5. Sardu C, D'Onofrio N, Balestrieri ML, Barbieri M, Rizzo MR, Messina V, et al. Hyperglycaemia on admission to hospital and COVID-19. Diabetologia. 2020; 63:2486-7.

6. Bode B, Garrett V, Messler J, McFarland R, Crowe J, Booth R, et al. Glycemic characteristics and clinical outcomes of COVID-19 patients hospitalized in the United States. J Diabetes Sci Technol. 2020;14:813-21.

7. Marfella R, Paolisso P, Sardu C, Bergamaschi L, D'Angelo EC, Barbieri M, et al. Negative impact of hyperglycaemia on tocilizumab therapy in Covid-19 patients. Diabetes Metab. 2020; S1262-3636(20)30080.3.
8. Pal R, Bhadada SK. Should anti-diabetic medications be reconsidered amid COVID-19 pandemic? Diabetes Res Clin Pract. 2020;163:108146.

9. Bornstein SR, Rubino F, Khunti K, Mingrone G, Hopkins D, Birkenfeld AL, et al. Practical recommendations for the management of diabetes in patients with COVID-19. Lancet Diabetes Endocrinol. 2020;8:546-50.

10. Ceriello A, Stoian AP, Rizzo M. COVID-19 and diabetes management: what should be considered? Diabetes Res Clin Pract. 2020;163:108151.

11. Apicella M, Campopiano MC, Mantuano M, Mazoni L, Coppelli A, Del Prato S. COVID-19 in people with diabetes: understanding the reasons for worse outcomes. Lancet Diabetes Endocrinol. 2020;8:782-92.

12. Chen Y, Yang D, Cheng B, Chen J, Peng A, Yang C, et al. Clinical characteristics and outcomes of patients with diabetes and COVID-19 in association with glucose-lowering medication. Diabetes Care. 2020;43:1399-407.

13. Bramante C, Ingraham N, Murray T, Marmor S, Hoversten S, Gronski J, et al. Observational study of metformin and risk of mortality in persons hospitalized with Covid-19. medRxiv. 2020; 06.19.20135095.

14. Rhee SY, Lee J, Nam H, Kyoung DS, Kim DJ. Effects of a DPP-4 inhibitor and RAS blockade on clinical outcomes of patients with diabetes and COVID-19. medRxiv. 2020; 05.20.20108555.

15. Hussain A, Bhowmik B, do Vale Moreira NC. COVID-19 and diabetes: knowledge in progress. Diabetes Res Clin Pract 2020;162:108142.

16. lacobellis G. COVID-19 and diabetes: can DPP4 inhibition play a role? Diabetes Res Clin Pract. 2020;162:108125.

17. Casas Rojo JM, Antón Santos JM, Millán Núñez-Cortés J, Lumbreras-Bermejo C, Ramos-Rincón JM, Roy-Vallejo E, et al. Clinical characteristics of patients hospitalized with COVID-19 in Spain: results from the SEMI-COVID-19 Network. Rev Clin Esp. 2020; 05.24.20111971.

18. Mahoney FI, Barthel DW. Functional evaluation: the Barthel Index. Md State Med J. 1965;14:61-5.

19. Charlson ME, Pompei $P$, Ales $K L$, MacKenzie CR. A new method of classifying prognostic comorbidity in longitudinal studies: development and validation. J Chronic Dis. 1987:40:373-83.

20. American Diabetes Association. Classification and diagnosis of diabetes: standards of medical care in diabetes-2020. Diabetes Care. 2020;43:S14-31.

21. Austin PC. Comparing paired vs non-paired statistical methods of analyses when making inferences about absolute risk reductions in propensity-score matched samples. Stat Med. 2011;30:1292-301.

22. Cameron AR, Morrison VL, Levin D, Mohan M, Forteath C, Beall C, et al. Antiinflammatory effects of metformin irrespective of diabetes status. Circ Res. 2016;119:652-65.

23. Costela-Ruiz VJ, Illescas-Montes R, Puerta-Puerta JM, Ruiz C, MelguizoRodríguez L. SARS-CoV-2 infection: the role of cytokines in COVID-19 disease. Cytokine Growth Factor Rev. 2020; S1359-6101(20)30109-X.

24. Berlin DA, Gulick RM, Martinez FJ. Severe Covid-19. N Engl J Med. 2020.

25. Rena G, Hardie DG, Pearson ER. The mechanisms of action of metformin. Diabetologia. 2017:60:1577-85.

26. Sharma S, Ray A, Sadasivam B. Metformin in COVID-19: a possible role beyond diabetes. Diabetes Res Clin Pract. 2020;164:108183.

27. Mehta P, McAuley DF, Brown M, Sanchez E, Tattersall RS, Manson JJ. COVID19: consider cytokine storm syndromes and immunosuppression. Lancet. 2020;395:1033-4.

28. Maddaloni E, Buzzetti R. Covid-19 and diabetes mellitus: unveiling the interaction of two pandemics. Diabetes Metab Res Rev. 2020:e33213321.

29. Drucker DJ. Coronavirus infections and type 2 diabetes-shared pathways with therapeutic implications. Endocr Rev. 2020;41(3):bnaa011.

30. Sardu C, Gambardella J, Morelli MB, Wang X, Marfella R, Santulli G. Hypertension, thrombosis, kidney failure, and diabetes: is COVID-19 an endothelial disease? A comprehensive evaluation of clinical and basic evidence. J Clin Med. 2020;9:1417.

31. Sardu C, Maggi P, Messina V, luliano P, Sardu A, lovinella V, et al. Could antihypertensive drug therapy affect the clinical prognosis of hypertensive patients with COVID-19 infection? Data from centers of southern Italy. J Am Heart Assoc. 2020;9:e016948.

32. Rodilla E, Saura A, Jiménez I, Mendizábal A, Pineda-Cantero A, LorenzoHernández $E$, et al. Association of hypertension with all-cause mortality among hospitalized patients with COVID-19. J Clin Med. 2020;9:E3136.

33. Cure E, Cumhur CM. Can dapagliflozin have a protective effect against COVID-19 infection? A hypothesis. Diabetes Metab Syndr. 2020;14:405-6.

\section{Publisher's Note}

Springer Nature remains neutral with regard to jurisdictional claims in published maps and institutional affiliations. 